Bull. Austral. Math. Soc.

$47 \mathrm{~B} 47,47 \mathrm{~L} 10$

VoL. 66 (2002) [227-232]

\title{
CHARACTERISATIONS OF DERIVATIONS ON SOME OPERATOR ALGEBRAS
}

\author{
Wu Jing, Shijie Lu and Pengtong Li
}

Some conditions under which a derivation on some operator algebras can be completely determined by the action on operators of zero product are given.

In recent years there has been considerable interest in studying which linear mappings on operator algebras are derivations $([1,5,6,7,8])$. It is the aim of this note to give conditions under which a derivation of some operator algebras can be completely determined by the action on operators of product-zero.

Before proceedings let us fix some notation. In what follows $X$ will be a (real or complex) Banach space. We denote by $B(X)$ the algebra of all bounded linear operators on $X$, and $F(X)$ denotes the algebra of all finite rank operators in $B(X)$. Recall that a standard operator algebra is any subalgebra of $B(X)$ which contains $F(X)$. The usual notation $L a t \mathcal{A}$ will denote the lattice of invariant subspaces for a subset $\mathcal{A} \subseteq B(X)$, and $\operatorname{Alg} \mathcal{L}$ will denote the algebra of bounded linear operators leaving invariant every member of a family $\mathcal{L}$ of subspaces. The subset $\mathcal{A}$ is reflexive if $\mathcal{A}=$ ref $\mathcal{A}$, where ref $\mathcal{A}=\{T \in B(X): T x \in[\mathcal{A} x], x \in X\}$ and [.] denotes the norm closure. A nest $\mathcal{N}$ in $X$ is a family of closed subspaces of $X$ totally ordered by inclusion containing $\{0\}$ and $X$, and the closed linear span and intersection of every subfamily. The associated nest algebra is $\operatorname{Alg} \mathcal{N}=\{T \in B(X): T N \subseteq N, N \in \mathcal{N}\}$.

For a lattice $\mathcal{L}$ of subspaces of $X$, if $N \in \mathcal{L}$, define $N_{-}=\vee\{M \in \mathcal{L}: N \nsubseteq M\}$ and $N_{+}=\wedge\{M \in \mathcal{L}: M \nsubseteq N\}$. We also define $0_{-}=0$ and $X_{+}=X$.

For a subset $N \subseteq X, N^{\perp}=\left\{f \in X^{*}: f(N)=\{0\}\right\}$, where $X^{*}$ is the dual space of $X$. If both $x \in X$ and $f \in X^{*}$ are nonzero, the rank one operator $u \longmapsto f(u) x$ is denoted by $x \otimes f$.

The following lemma will get repeated use.

LEMMA 1. ([9]) If $\mathcal{L}$ is a subspace lattice, then $x \otimes f \in \operatorname{Alg} \mathcal{L}$ if and only if there exists an element $L \in \mathcal{L}$ such that $x \in L$ and $f \in\left(L_{-}\right)^{\perp}$.

Let us begin with two key lemmas.

Lemma 2. Let $\mathcal{A}$ be an algebra containing identity $I$ and $\delta: \mathcal{A} \rightarrow \mathcal{A}$ be a linear map with the property that $\delta(A B)=\delta(A) B+A \delta(B)$ holds for every pair $A, B \in \mathcal{A}$ with $A B=0$. Then for each idempotent $P \in \mathcal{A}$, we have

Received 3rd January, 2002

Copyright Clearance Centre, Inc. Serial-fee code: 0004-9727/02 \$A2.00+0.00. 
(1) $P \delta(I)=\delta(I) P=P \delta(I) P=P \delta(P) P$ and

(2) $\delta\left(P^{2}\right)=\delta(P) P+P \delta(P)-P \delta(I) P$.

Proof: For any idempotent $P \in \mathcal{A}$, it is obvious that $P(I-P)=(I-P) P=0$. -Then we have

(1) $0=\delta(P(I-P))=\delta(P)(I-P)+P \delta(I-P)=\delta(P)-\delta(P) P+P \delta(I)-P \delta(P)$

and

(2) $0=\delta((I-P) P)=\delta(I-P) P+(I-P) \delta(P)=\delta(I) P-\delta(P) P+\delta(P)-P \delta(P)$.

Comparing equalities (1) and (2), we arrive at

$$
P \delta(I)=\delta(I) P
$$

Since $P$ is idempotent, then we get $P \delta(I)=\delta(I) P=P \delta(I) P$.

Multiplying equation (1) by $P$ from the left, we have $P \delta(P) P=P \delta(I)$.

Now equality (1) becomes

$$
\delta\left(P^{2}\right)=\delta(P)=\delta(P) P+P \delta(P)-P \delta(I)=\delta(P) P+P \delta(P)-P \delta(I) P .
$$

Lemma 3. Suppose that $\mathcal{A}$ and $\delta$ are the same as in Lemma 2. Then for any idempotent $P$ in $\mathcal{A}$ and arbitrary $A \in \mathcal{A}$, we have

(1) $\delta(P A)=\delta(P) A+P \delta(A)-P \delta(I) A$ and

(2) $\delta(A P)=\delta(A) P+A \delta(P)-A \delta(I) P$.

Proof: We only prove (1), and (2) goes similarly.

Obviously, $P(I-P) A=(I-P) P A=0$. Then we have

$$
\begin{aligned}
0 & =\delta(P(I-P) A) \\
& =\delta(P)(A-P A)+P \delta(A-P A) \\
& =\delta(P) A-\delta(P) P A+P \delta(A)-P \delta(P A)
\end{aligned}
$$

and

$$
\begin{aligned}
0 & =\delta((I-P) P A) \\
& =\delta(I-P) P A+(I-P) \delta(P A) \\
& =\delta(I) P A-\delta(P) P A+\delta(P A)-P \delta(P A)
\end{aligned}
$$

Comparing (3) and (4), and using Lemma 2, we obtain

$$
\delta(P A)=\delta(P) A+P \delta(A)-P \delta(I) A .
$$

Now we are in a position to prove our main results. 
THEOREM 4. Let $\mathcal{M}$ be a von Neumann algebra in Hilbert space $H$, and $\delta: \mathcal{M}$ $\rightarrow \mathcal{M}$ be a linear map for which $\delta(A B)=\delta(A) B+A \delta(B)$ holds for any pair $A, B \in \mathcal{M}$ with $A B=0$. If $\delta$ is norm continuous, then $\delta(A B)=\delta(A) B+A \delta(B)-A \delta(I) B$ holds for every $A, B \in \mathcal{M}$. Particularly, if $\delta$ satisfies $\delta(I)=0$ additionally, then $\delta$ is a derivation.

Proof: We notice that the linear span of all projections (that is, self-adjoint idempotents) of $\mathcal{M}$ is norm dense in $\mathcal{M}$ (see [2]). By Lemma 3, the theorem goes easily.

THEOREM 5. Let Alg $\mathcal{N}$ be a nest algebra in Hilbert space $H$. Suppose that $\delta$ : $\operatorname{Alg} \mathcal{N} \rightarrow \operatorname{Alg} \mathcal{N}$ is a linear map with the property that $\delta(A B)=\delta(A) B+A \delta(B)$ holds for any pair $A, B \in \operatorname{Alg} \mathcal{N}$ with $A B=0$. Then for arbitrary $A$ and $B$ in $\operatorname{Alg} \mathcal{N}$, we have $\delta(A B)=\delta(A) B+A \delta(B)-A \delta(I) B$. Furthermore, if $\delta(I)=0$ additionally, then $\delta$ is a derivation.

PROOF: Notice that every rank one operator in $\operatorname{Alg} \mathcal{N}$ can be represented as a linear combination of at most four idempotents in $A \lg \mathcal{N}$ (see [4]) and every finite rank operator in $\operatorname{Alg} \mathcal{N}$ can be written as a finite sum of rank one operators in $\operatorname{Alg} \mathcal{N}$ (see [3]). Then by Lemma 3, we get that for arbitrary $A \in \operatorname{Alg} \mathcal{N}$ and any finite rank operator $F \in \operatorname{Alg} \mathcal{N}$

$$
\delta(A F)=\delta(A) F+A \delta(F)-A \delta(I) F
$$

Now, for any pair $A, B \in \operatorname{Alg} \mathcal{N}$ and any finite rank operator $F \in \operatorname{Alg} \mathcal{N}$, on the one hand, we have

$$
\delta(A \dot{B} F)=\delta(A B) F+A B \delta(F)-A B \delta(I) F
$$

On the other hand,

$$
\begin{aligned}
\delta(A B F) & =\delta(A) B F+A \delta(B F)-A \delta(I) B F \\
& =\delta(A) B F+A \delta(B) F+A B \delta(F)-A B \delta(I) F-A \delta(I) B F
\end{aligned}
$$

These two expressions imply

$$
[\delta(A B)-(\delta(A) B+A \delta(B)-A \delta(I) B)] F=0 .
$$

Therefore (since $\cup\left\{N \in \mathcal{N}: N_{-} \neq H\right\}$ is dense in $\left.H\right) \delta(A B)=\delta(A) B+A \delta(B)$ - $A \delta(I) B$.

Now we turn our attention to the algebras in Banach spaces.

THEOREM 6. Let $\mathcal{B}$ be a standard operator algebra in Banach space $X$ containing the identity operator $I$, and $\delta: \mathcal{B} \rightarrow \mathcal{B}$ be a linear map such that $\delta(A B)=\delta(A) B+A \delta(B)$ for any pair $A, B \in \mathcal{B}$ with $A B=0$. Then $\delta(A B)=\delta(A) B+A \delta(B)-A \delta(I) B, \forall A, B \in \mathcal{B}$. Moreover, if in addition $\delta(I)=0$, then $\delta$ is a derivation.

Proof: Since every rank one operator in $F(X)$ can be represented as a linear combination of at most two rank one idempotents and every finite rank operator in $F(X)$ is a finite sum of rank one operators, we get that $\delta(A F)=\delta(A) F+A \delta(F)-A \delta(I) F$ 
holds for any $A \in \mathcal{B}$ and any $F \in F(X)$. With the same argument as in the proof of Theorem 5 , we obtain

$$
\delta(A B)=\delta(A) B+A \delta(B)-A \delta(I) B
$$

for every $A, B \in \mathcal{B}$.

Unfortunately, whether every rank one operator in a nest algebra $\operatorname{Alg} \mathcal{N}$ in Banach space $X$ can be represented as a finite linear combination of idempotents in $\operatorname{Alg} \mathcal{N}$ is uncertain. So Theorem 5 can't be generalised to the Banach space case. But we do have the following theorem.

THEOREM 7. Let $\mathcal{R}$ be a unital reflexive algebra in Banach space $X$ such that $0_{+} \neq 0$ or $X_{-} \neq X$ in LatR. Suppose that $\delta: \mathcal{R} \rightarrow \mathcal{R}$ is a linear mapping satisfying $\delta(A B)=\delta(A) B+A \delta(B)$ for any pair $A, B \in \mathcal{R}$ with $A B=0$. Then $\delta(A B)=\delta(A) B+$ $A \delta(B)-A \delta(I) B$ holds for arbitrary $A$ and $B$ in $\mathcal{R}$. Furthermore, if $\delta(I)=0$ additionally, then $\delta$ is a derivation.

Proof: Suppose that $X_{-} \neq X$.

We claim that $\delta(A F)=\delta(A) F+A \delta(F)-A \delta(I) F$ holds for any $A \in \mathcal{R}$ and any rank one operator $F \in \mathcal{R}$ of the form $F=x \otimes f$ with $x \in X$ and $f \in\left(X_{-}\right)^{\perp}$.

We only need to treat the case where $f(x)=0$ since, otherwise, $F$ is a scalar multiple of an idempotent. Suppose that $f(x)=0$.

We can choose $y \in X$ such that $f(y)=1$. Then $y \otimes f \in \mathcal{R}$, and both $(x+y) \otimes f$ and $y \otimes f$ are idempotent. By Lemma 3, we have

$$
\delta(A(x+y) \otimes f)=\delta(A)(x+y) \otimes f+A \delta((x+y) \otimes f)-A \delta(I)(x+y) \otimes f
$$

and

$$
\delta(A y \otimes f)=\delta(A) y \otimes f+A \delta(y \otimes f)-A \delta(I) y \otimes f .
$$

Hence $\delta(A x \otimes f)=\delta(A) x \otimes f+A \delta(x \otimes f)-A \delta(I) x \otimes f$.

The argument in the preceding paragraph works even if $x \notin X_{-}$.

Therefore for any pair $A, B \in \mathcal{R}$ and any $x \in X, f \in\left(X_{-}\right)^{\perp}$, we have

$$
\delta(A B x \otimes f)=\delta(A B) x \otimes f+A B \delta(x \otimes f)-A B \delta(I) x \otimes f
$$

and

$$
\begin{aligned}
\delta(A B x \otimes f)= & \delta(A) B x \otimes f+A \delta(B x \otimes f)-A \delta(I) B x \otimes f \\
= & \delta(A) B x \otimes f+ \\
& -A \delta(B) x \otimes f+A B \delta(x \otimes f) \\
& -A B \delta(I) x \otimes f-A \delta(I) B x \otimes f .
\end{aligned}
$$

These two equalities yield

$$
[\delta(A B)-(\delta(A) B+A \delta(B)-A \delta(I) B)] x \otimes f=0 .
$$


This is true for every $x \in X$, so

$$
\delta(A B)=\delta(A) B+A \delta(B)-A \delta(I) B
$$

for every $A, B \in \mathcal{R}$.

For the case of $0_{+} \neq 0$, using equation $\delta(P A)=\delta(P) A+P \delta(A)-P \delta(I) A$ in Lemma 3 and an argument similar to the one above, we can infer that

$$
x \otimes f[\delta(A B)-(\delta(A) B+A \delta(B)-A \delta(I) B)]=0
$$

for any $x \in 0_{+}, f \in X^{*}$ and any $A, B \in \mathcal{R}$. Since $f \in X^{*}$ is arbitrary, thus for any pair $A, B \in \mathcal{R}$ we have

$$
\delta(A B)=\delta(A) B+A \delta(B)-A \delta(I) B .
$$

Particularly, we can generalise Theorem 5 partially as follows.

THEOREM 8. Let $\mathrm{Alg} \mathcal{N}$ be a nest algebra in Banach space $X$ such that $0_{+} \neq 0$ or $X_{-} \neq X$ in $\mathcal{N}$. Suppose that $\delta: \operatorname{Alg} \mathcal{N} \rightarrow \operatorname{Alg} \mathcal{N}$ is a linear mapping with the property that $\delta(A B)=\delta(A) B+A \delta(B)$ holds for any pair $A, B \in \operatorname{Alg} \mathcal{N}$ with $A B=0$. Then for arbitrary $A$ and $B$ in $\operatorname{Alg} \mathcal{N}$ we have $\delta(A B)=\delta(A) B+A \delta(B)-A \delta(I) B$. Moreover, if $\delta(I)=0$ additionally, then $\delta$ is a derivation.

REMARK 9. It should be mentioned that $\delta(I)$ in Theorems $4,5,6,7$ and 8 is in the commutants of the associated algebras $\mathcal{M}, \operatorname{Alg} \mathcal{N}, \mathcal{B}, \mathcal{R}$ and $\operatorname{Alg} \mathcal{N}$ respectively. For example, let $\delta$ and $\mathcal{R}$ be as in Theorem 7 , and suppose that $X_{-} \neq X$ in Lat $\mathcal{R}$. By Lemma 2 and with the similar argument in the proof of Theorem 7, we can infer that $\delta(I) x \otimes f=x \otimes f \delta(I)$ holds for any $x \in X$ and $f \in\left(X_{-}\right)^{\perp}$. Then for arbitrary $A \in \mathcal{R}$ we have $(\delta(I) A) x \otimes f=\delta(I)(A x \otimes f)=(A x \otimes f) \delta(I)=A(x \otimes f \delta(I))=A(\delta(I) x \otimes f)$ $=(A \delta(I)) x \otimes f$. This is true for all $x \in X$, hence $\delta(I) A=A \delta(I)$, that is $\delta(I) \in \mathcal{R}^{\prime}$, the commutant of $\mathcal{R}$. Thus the result $\delta(A B)=\delta(A) B+A \delta(B)-A \delta(I) B$ coincides with the condition that $\delta(A B)=\delta(A) B+A \delta(B)$ when $A B=0$.

\section{REFERENCES}

[1] M. Brešar and P. Šemrl, 'Mappings which preserve idempotents, local automorphisms and local derivations', Canad. J. Math. 45 (1993), 483-498.

[2] J. Dixmier, von Neumann algebras (North-Holland Publishing Company, Amsterdam, New York, 1981).

[3] J.A. Erdos, 'Operators of finite rank in nest algebras', J. London Math. Soc. 43 (1968), 391-397.

[4] L.B. Hadwin, 'Local multiplications on algebras spanned by idempotents', Linear and Multilinear Algebra 37 (1994), 259-263.

[5] W. Jing, 'Local derivations of reflexive algebras', Proc. Amer. Math. Soc. 125 (1997), 869-873. 
[6] W. Jing, 'Local derivations of reflexive algebras II', Proc. Amer. Math. Soc. 129 (2001), 1933-1937.

[7] R.V. Kadison, 'Local derivations', J. Algebra 130 (1990), 495-509.

[8] D.R. Larson and A.R. Sourour, 'Local derivations and local automorphisms of $B(X)$ ', Proc. Sympos. Pure. Math. 51 (1990), 187-194.

[9] W.E. Longstaff, 'Strongly reflexive lattices', J. London Math. Soc. 11 (1975), 491-498.

Department of Mathematics

Yuquan Campus

Zhejiang University

Hangzhou 310027

People's Republic of China

e-mail: jingwu@math.zju.edu.cn
Department of Mathematics

Yuquan Campus

Zhejiang University

Hangzhou 310027

People's Republic of China

e-mail: lusj@zucc.edu.cn

Department of Mathematics

Nanjing University

Nanjing 210093

People's Republic of China

e-mail: ptli@nju.edu.cn 